\title{
Quotients of Continuous Convex Functions on Nonreflexive Banach Spaces
}

\author{
by \\ P. HOLICKÝ, O. F. K. KALENDA, \\ L. VESELÝ and L. ZAJÍČEK \\ Presented by Aleksander PEŁCZYŃSKI
}

Summary. On each nonreflexive Banach space $X$ there exists a positive continuous convex function $f$ such that $1 / f$ is not a d.c. function (i.e., a difference of two continuous convex functions). This result together with known ones implies that $X$ is reflexive if and only if each everywhere defined quotient of two continuous convex functions is a d.c. function. Our construction also gives a stronger version of Klee's result concerning renormings of nonreflexive spaces and non-norm-attaining functionals.

A function on a Banach space $X$ is called a d.c. function if it can be represented as a difference of two continuous convex functions (all functions considered in this note are real-valued). Thus the system of all d.c. functions on $X$ is the smallest vector space containing all continuous convex functions. Moreover, it is well known (see, e.g., [3, III.2]), and not difficult to show, that it is also closed with respect to taking products and pointwise maxima; hence it is even an algebra and a lattice. While an everywhere defined quotient $g / f$ of two d.c. functions on a finite-dimensional Banach space is always d.c. (cf. [2, Corollary]), the situation is completely different for infinite-dimensional spaces: by [7, Corollary 5.6], on each infinitedimensional Banach space there exists a positive d.c. function such that $1 / f$ is not d.c.

2000 Mathematics Subject Classification: 46B10, 46B03.

Key words and phrases: reflexivity, d.c. functions, non-norm-attaining functionals, renormings.

The third author was supported in part by the Ministero dell'Università e della Ricerca of Italy. The other authors were supported by MSM 0021620839 financed by MSMT of Czech Republic, by GAČR 201/06/0198 and GAČR 201/06/0018. 
The following natural question arises:

Is the quotient $g / f$ of two continuous convex functions on $X$ d.c. if $f \neq 0$ ? Quite surprisingly, the answer is affirmative for all reflexive spaces $X$; indeed, it is proved in [7, Remark 3.5(i)] that $1 / f(f \neq 0$ continuous and convex $)$ is d.c. on $X$ whenever $X$ is reflexive. The main aim of this note is to show that the above question has a negative answer for each nonreflexive Banach space $X$.

The following criterion for non-d.c. functions (cf. [7, Lemma 5.1]) suggests how to construct a counterexample.

Lemma 1. Let $X$ be a Banach space and $h: X \rightarrow \mathbb{R}$ be a function. If there exist sets $M \subset X$ of arbitrarily small diameter such that $h$ is unbounded on $M$, then $h$ is not a d.c. function.

If there exists a continuous convex function $f$ on $X$ such that

(1) $\quad f>0$, and there exist sets $M$ of arbitrarily small diameters with $\inf f(M)=0$,

then $1 / f$ is not a d.c. function by Lemma 1. (Of course, such an $f$ cannot exist if $X$ is reflexive since, in this case, $f$ attains its minimum on any closed ball.)

To construct $f$, it might seem natural to proceed by finding an $x^{*} \in X^{*}$ such that

$x^{*}$ does not attain its norm, and there exist sets $M \subset B_{X}$ of arbitrarily small diameter such that $\sup x^{*}(M)=\left\|x^{*}\right\|_{*}$.

Indeed, if we had such an $x^{*}$, it would be sufficient to put $f(x):=\|x\|-\left\|x^{*}\right\|_{*}$ if $x^{*}(x)=\left\|x^{*}\right\|_{*}$, and to extend $f$ to the whole $X$ so that $f$ is constant on each line parallel to a fixed vector $v \in X$ such that $x^{*}(v) \neq 0$. While it is not difficult to check that no such $x^{*}$ exists in the classical nonreflexive spaces $c_{0}$ and $\ell_{1}$ (with their canonical norms), it is possible to prove (see below) that such an $x^{*}$ always exists after a suitable equivalent renorming of (a nonreflexive) $X$.

However, we proceed in a different order. First, using James' sequential characterization of nonreflexivity, we construct a continuous convex function $f$ on $X$, satisfying (1), as a distance function from a certain bounded convex set in $X \oplus \mathbb{R}$. Using this $f$, we easily prove our main Theorem 4 , which also gives a modification of the well known characterization of nonreflexive spaces by monotone sequences of closed convex sets. Then, using the existence of such $f$ on each hyperplane of $X$, we show that, if $X$ is nonreflexive, each nonzero functional $x^{*} \in X^{*}$ satisfies (2) with respect to a suitable equivalent norm on $X$. This last assertion is the content of Proposition 5 which we believe to be of independent interest since it improves the following result 
of Klee [5]: each nonzero bounded linear functional on a nonreflexive Banach space $X$ is non-norm-attaining for some equivalent norm on $X$.

Let us start by fixing some notations. We consider only Banach spaces over the reals. We denote by $B_{X}$ or $B_{(X,\|\cdot\|)}$ the closed unit ball in a Banach space $X$ endowed with a norm $\|\cdot\|$. By $\|\cdot\|_{*}$ we denote the corresponding dual norm on $X^{*}$ (the topological dual of $X$ ).

In what follows, we consider $X \oplus \mathbb{R}$ equipped with the maximum norm, and we identify $x \in X$ with $(x, 0) \in X \oplus \mathbb{R}$ (and so $X$ with $X \times\{0\}$ ).

Lemma 2. Let $X$ be a nonreflexive Banach space. Then there exists a nonempty bounded convex set $C \subset X \oplus \mathbb{R}$ such that

(a) $\varphi(x):=\operatorname{dist}(x, C)>0$ for every $x \in X$,

(b) for each $\varepsilon>0$ there is a set $M_{\varepsilon} \subset X$ with $\operatorname{diam} M_{\varepsilon}<\varepsilon$ and $\inf \varphi\left(M_{\varepsilon}\right)=0$.

Proof. Since $X$ is nonreflexive, by [4, Theorem 1] (see, e.g., [1, Theorem 10.3] or [6, Theorem 1.13.4] for simpler proofs) there exist unit vectors $\left\{e_{i}\right\}_{i=1}^{\infty}$ in $X$ and unit functionals $\left\{e_{i}^{*}\right\}_{i=1}^{\infty}$ in $X^{*}$ such that

$$
e_{i}^{*}\left(e_{j}\right)=0 \quad \text { if } i>j, \quad e_{i}^{*}\left(e_{j}\right)>1 / 2 \quad \text { if } i \leq j .
$$

Set $e_{\infty}:=(0,1) \in X \oplus \mathbb{R}$, and let $f_{i} \in(X \oplus \mathbb{R})^{*}$ be the extension of $e_{i}^{*}$ for which $f_{i}\left(e_{\infty}\right)=1$. Clearly $\left\|f_{i}\right\|_{*}=2$. For $0<k<n$ in $\mathbb{N}$, we define

$$
x_{k, n}:=2 e_{k}+\frac{2}{k} e_{n}+\frac{1}{n} e_{\infty} .
$$

Clearly

$$
\begin{array}{ll}
f_{i}\left(x_{k, n}\right) \geq 1 & \text { for } 1 \leq i \leq k, \\
f_{i}\left(x_{k, n}\right) \geq \frac{1}{k} & \text { for } k<i \leq n, \\
f_{i}\left(x_{k, n}\right)=\frac{1}{n} & \text { for } i>n .
\end{array}
$$

We define

$$
C:=\operatorname{conv}\left\{x_{k, n}: 0<k<n, k, n \in \mathbb{N}\right\}, \quad X_{0}:=\overline{\operatorname{span}}\left\{e_{j}: j \in \mathbb{N}\right\} .
$$

To prove (a), we need to show $\bar{C} \cap X=\emptyset$. Since clearly $\bar{C} \cap X \subset X_{0}$, it is sufficient to show that $\bar{C} \cap X_{0}=\emptyset$. So, suppose to the contrary that an $x_{0} \in \bar{C} \cap X_{0}$ is given. As $\left\|f_{i}\right\|_{*}=2$ and $\lim _{i \rightarrow \infty} f_{i}\left(e_{j}\right)=0$ for each $j \in \mathbb{N}$, it is easy to check that $\lim _{i \rightarrow \infty} f_{i}(x)=0$ for every $x \in X_{0}$. So, we may find natural numbers $i_{1}<i_{2}<i_{3}$ such that

$$
f_{i_{1}}\left(x_{0}\right)<\frac{1}{3}, \quad i_{1} f_{i_{2}}\left(x_{0}\right)<\frac{1}{3}, \quad i_{2} f_{i_{3}}\left(x_{0}\right)<\frac{1}{3} .
$$

Since $x_{0} \in \bar{C}$ and $f_{i_{1}}, f_{i_{2}}, f_{i_{3}}$ are continuous, we can find $c \in C$ so close to 
$x_{0}$ that

$$
f_{i_{1}}(c)<\frac{1}{3}, \quad i_{1} f_{i_{2}}(c)<\frac{1}{3}, \quad i_{2} f_{i_{3}}(c)<\frac{1}{3} .
$$

Since $c \in C$, we can assign to each $(k, n)$ with $1 \leq k<n$ a number $\alpha_{k, n} \geq 0$ so that $\sum \alpha_{k, n}=1$, the set $\left\{(k, n): \alpha_{k, n} \neq 0\right\}$ is finite, and $c=\sum \alpha_{k, n} x_{k, n}$.

Using (4), (5), and (6) in turn, we obtain

$$
\begin{aligned}
f_{i_{1}}(c)=\sum \alpha_{k, n} f_{i_{1}}\left(x_{k, n}\right) \geq \sum_{\substack{k \geq i_{1} \\
n>k}} \alpha_{k, n}, \\
f_{i_{2}}(c)=\sum \alpha_{k, n} f_{i_{2}}\left(x_{k, n}\right) \geq \sum_{\substack{k<i_{1} \\
n \geq i_{2}}} \frac{1}{k} \alpha_{k, n} \geq \frac{1}{i_{1}} \sum_{\substack{k<i_{1} \\
n \geq i_{2}}} \alpha_{k, n}, \\
f_{i_{3}}(c)=\sum \alpha_{k, n} f_{i_{3}}\left(x_{k, n}\right) \geq \sum_{\substack{k<i_{1} \\
n<i_{2}}} \frac{1}{n} \alpha_{k, n} \geq \frac{1}{i_{2}} \sum_{\substack{k<i_{1} \\
n<i_{2}}} \alpha_{k, n} .
\end{aligned}
$$

Using (9), (10), (11) and (8), we easily obtain $\sum \alpha_{k, n}<1$, which is a contradiction.

To prove (b), consider an arbitrary $\varepsilon>0$. Choose $k_{0} \in \mathbb{N}$ with $4 / k_{0}<\varepsilon$ and set $M_{\varepsilon}:=\left\{2 e_{k_{0}}+\left(2 / k_{0}\right) e_{n}: n>k_{0}\right\}$. Then clearly diam $M_{\varepsilon} \leq 4 / k_{0}<\varepsilon$. The other desired property of $M_{\varepsilon}$ also holds, since, for each $n>k_{0}$,

$$
\begin{aligned}
\inf \varphi\left(M_{\varepsilon}\right) & =\operatorname{dist}\left(M_{\varepsilon}, C\right) \\
& \leq\left\|\left(2 e_{k_{0}}+\left(2 / k_{0}\right) e_{n}\right)-\left(2 e_{k_{0}}+\left(2 / k_{0}\right) e_{n}+(1 / n) e_{\infty}\right)\right\|=1 / n
\end{aligned}
$$

\section{REMARK 3.}

(i) To obtain $C$ with the weaker property $\inf _{x \in X} \varphi(x)=0$ instead of (b) in Lemma 2 , it is sufficient to put $C:=\operatorname{conv}\left\{2 e_{k}+(1 / k) e_{\infty}: k \in \mathbb{N}\right\}$, and the proof becomes simpler.

(ii) Set $C:=\operatorname{conv}\left\{2 e_{k}+(2 / k) e_{n}+(2 / n) e_{m}+(1 / m) e_{\infty}: 0<k<n<m\right.$, $k, n, m \in \mathbb{N}\}$. An easy modification of the proof of Lemma 2 gives the following property which is slightly stronger than (b):

$\left(\mathrm{b}^{2}\right)$ there exist sets $M \subset X$ of arbitrarily small diameter such that $M$ contains sets $A$ of arbitrarily small diameter with inf $\varphi(A)=0$.

(Analogously, using indices $0<k_{1}<\cdots<k_{p+1}$ in the definition of $C$, it is possible to obtain the corresponding iterated property $\left(\mathrm{b}^{p}\right)$.)

Now, we are ready to state the following main result of the present paper.

THEOREM 4. The following properties of a Banach space $X$ are equivalent. 
(a) $X$ is nonreflexive.

(b) There is a continuous convex function $f: X \rightarrow(0, \infty)$ such that $1 / f$ is not representable as a difference of two continuous convex functions.

(c) There is a decreasing sequence $\left\{C_{n}\right\}_{n=1}^{\infty}$ of bounded closed convex subsets of $X$ such that

$$
\bigcap_{n=1}^{\infty} C_{n}=\emptyset, \quad \bigcap_{n=1}^{\infty}\left(C_{n}+\varepsilon B_{X}\right) \neq \emptyset \quad \text { for every } \varepsilon>0 .
$$

Proof. If $X$ is nonreflexive, take $f:=\varphi$ where $\varphi$ is as in Lemma 2. By Lemma $1,1 / f$ is not d.c. on $X$. On the other hand, if $X$ is reflexive and $f$ is a positive continuous convex function, then $1 / f$ is d.c. on $X$ by $[7, \operatorname{Re}-$ mark 3.5(i)]. Thus (a) and (b) are equivalent.

Let us show that (a) and (c) are equivalent. If $X$ is nonreflexive, let $\varphi$ be again the function from Lemma 2. The sets $C_{n}:=\{x \in X: \varphi(x) \leq 1 / n\}$, $n \in \mathbb{N}$, are nonempty, closed, convex, bounded (since the set $C$ in Lemma 2 is bounded) and their intersection is empty. Let $\varepsilon>0$. By the properties of $\varphi$, there exists $x \in X$ such that, for each $n$, there is $y \in B(x, \varepsilon)$ with $\varphi(y) \leq 1 / n$, i.e. $y \in C_{n}$. In other words, $x \in \bigcap_{n=1}^{\infty}\left(C_{n}+\varepsilon B_{X}\right)$. Hence (a) implies (c). On the other hand, if $X$ is reflexive, then each decreasing sequence $\left\{C_{n}\right\}$ of nonempty closed bounded convex subsets of $X$ has a nonempty intersection, since each $C_{n}$ is weakly compact.

Let us conclude our paper with the promised strengthening of a result from [5].

Proposition 5. Let $Y$ be a nonreflexive Banach space and $0 \neq y^{*} \in Y^{*}$. Then there exists an equivalent norm $|\cdot|$ on $Y$ such that

(a) $y^{*}$ does not attain its norm on $B_{(Y,|\cdot|)}$,

(b) for each $\varepsilon>0$, there is $M_{\varepsilon} \subset B_{(Y,|\cdot|)}$ such that $\operatorname{diam} M_{\varepsilon}<\varepsilon$ and $\sup y^{*}\left(M_{\varepsilon}\right)=\left|y^{*}\right|_{*}$.

Proof. Set $X:=\left\{y \in Y: y^{*}(y)=0\right\}$ and choose $e \in Y$ with $y^{*}(e)=1$. Up to renorming, we may suppose that the norm on $Y$ satisfies

$$
\|y\|=\max \left\{\left\|y-y^{*}(y) e\right\|,\left|y^{*}(y)\right|\right\} \quad \text { for all } y \in Y .
$$

In this way we may identify $Y$ with $X \oplus_{\infty} \mathbb{R}$ so that $y^{*}((x, t))=t$ for $(x, t) \in X \times \mathbb{R}$.

As $Y$ is not reflexive, neither is $X$. Let $\varphi$ be the function on $X$ given by Lemma 2. Choose $\alpha>\varphi(0)$ and set

$$
A=\{x \in X: \varphi(x)<\alpha\} .
$$

By the properties of $\varphi$ the set $A$ is bounded. Therefore we can choose $r>0$ such that $A \subset B(0, r)$. Choose $\beta>\sup \varphi(B(0, r))$; this is possible as $\varphi$ is 
1-Lipschitz. Further, define

$$
\begin{aligned}
& D=\{(x, t) \in X \times \mathbb{R}: x \in B(0, r), t=\varphi(x)-\beta\}, \\
& C=\overline{\operatorname{conv}}(D \cup(-D)) .
\end{aligned}
$$

Then $C$ is clearly a bounded closed convex symmetric set. Further, $0 \in \operatorname{int} C$, as $0 \in A$ and $A \times(\alpha-\beta, \beta-\alpha) \subset C$. It follows that there exists an equivalent norm $|\cdot|$ on $X \times \mathbb{R}$ such that $C$ is the closed unit ball in this norm. We will show that this norm has the required properties.

We have

$$
\begin{aligned}
-\left|y^{*}\right|_{*} & =\inf y^{*}(C)=\inf y^{*}(D \cup(-D))=\inf y^{*}(D) \\
& =\inf \{\varphi(x)-\beta: x \in B(0, r)\}=-\beta
\end{aligned}
$$

as clearly inf $\varphi(B(0, r))=\inf \varphi(X)=0$. Thus $\left|y^{*}\right|_{*}=\beta$.

Next we show that $y^{*}$ does not attain its norm on $C$. Suppose it does. Then there is a point $z=\left(x_{0},-\beta\right) \in C$ (recall that $y^{*}((x, t))=t$ ). Note that

$$
C \subset\{(x, t) \in X \times \mathbb{R}: x \in B(0, r) \& t \geq \varphi(x)-\beta\} .
$$

The reason is that the set on the right hand side is closed and convex and it contains both $D$ and $-D$. It follows that $z$ belongs to the set on the right hand side, i.e. $-\beta \geq \varphi\left(x_{0}\right)-\beta$. So $\varphi\left(x_{0}\right) \leq 0$, a contradiction.

It remains to show (b). Let $\varepsilon>0$ be given. By the properties of $\varphi$ we can choose a set $P_{\varepsilon} \subset A$ such that $\operatorname{diam} P_{\varepsilon}<\varepsilon$ and $\inf \varphi\left(P_{\varepsilon}\right)=0$. (Note that $\varphi \geq \alpha$ outside $A$.) Now set

$$
P_{\varepsilon}^{*}:=\left\{(x, t) \in X \times \mathbb{R}: x \in P_{\varepsilon}, t=\varphi(x)-\beta\right\} .
$$

Then clearly $P_{\varepsilon}^{*} \subset C$ and

$$
\inf _{z \in P_{\varepsilon}^{*}} y^{*}(z)=-\beta=-\left|y^{*}\right|_{*} \cdot
$$

As $\varphi$ is 1 -Lipschitz with respect to $\|\cdot\|$, we see that $\|\cdot\|$-diam $P_{\varepsilon}^{*}<\varepsilon$. Set $M_{\varepsilon}:=-P_{\varepsilon / K}^{*}$, where $K>0$ is such that $|\cdot| \leq K\|\cdot\|$ on $X \times \mathbb{R}$. Then $M_{\varepsilon}$ has all required properties and the proof is complete.

\section{References}

[1] D. van Dulst, Reflexive and Superreflexive Banach Spaces, Math. Centre Tracts 102, Math. Centrum, Amsterdam, 1978.

[2] P. Hartman, On functions representable as a difference of convex functions, Pacific J. Math. 9 (1959), 707-713.

[3] J.-B. Hiriart-Urruty, Generalized differentiability, duality and optimization for problems dealing with differences of convex functions, in: Convexity and Duality in Optimization (Groningen, 1984), Lecture Notes in Econom. and Math. Systems 256, Springer, Berlin, 1985, 37-70.

[4] R. C. James, Characterizations of reflexivity, Studia Math. 23 (1964), 205-216.

[5] V. L. Klee, Jr., Some characterizations of reflexivity, Rev. Ci. Lima 52 (1950), 15-23. 
[6] R. E. Megginson, An Introduction to Banach Space Theory, Grad. Texts in Math. 183, Springer, New York, 1998.

[7] L. Veselý and L. Zajíček, On compositions of delta-convex mappings and functions, preprint, http://arxiv.org/abs/0706.0624, 2007.

P. Holický, O. F. K. Kalenda and L. Zajíček

Faculty of Mathematics and Physics

Charles University

Sokolovská 83

18675 Praha 8, Czech Republic

E-mail: holicky@karlin.mff.cuni.cz

kalenda@karlin.mff.cuni.cz

zajicek@karlin.mff.cuni.cz

L. Veselý

Dipartimento di Matematica "F. Enriques"

Università degli Studi di Milano

Via C. Saldini 50

20133 Milano, Italy

E-mail: vesely@mat.unimi.it

Received June 6, 2007;

received in final form September 3, 2007 